\title{
SYMPOSIUM EXPERIMENTAL APPROACHES TO EATING AND ITS DISORDERS
}

\author{
Held at the 31st Annual Meeting of the Psychonomic Society \\ November 1990, New Orleans, Louisiana \\ Chaired by Elizabeth D. Capaldi, University of Florida
}

The topic of this symposium has tremendous practical importance. Over 30 million Americans are obese (and over 64 million are trying to lose weight), and eating disorders are increasingly prevalent. All areas of experimental psychology are relevant to the understanding of eating and its disorders, and the symposium brought together leading researchers from a number of different areas who work on this topic.

The earliest work in experimental psychology on feeding was done by physiological psychologists who were interested in identifying the internal cues that regulate feeding, primarily by using a negative feedback homeostatic model. A great deal of progress has been made toward understanding the biological factors underlying eating; Neal Rowland reviews the data. At the same time, it has become clear that animals, including humans, eat both in anticipation of their needs and for sensory pleasure. Linda Bartoshuk reviews the role of sensory factors in ingestion. Diet and food selection are now seen as critical factors in obesity and learning, and cognitive factors are primary determinants of food preferences. In animal learning, there has recently been considerable interest in how food preferences are learned; this began in the 1960s with the discovery of taste aversion learning. This robust learning phenomenon seemed to differ in important ways from other forms of learning, which lead to an explosion of research on the topic. More recent research has also focused on taste preferences learned on the basis of positive outcomes, taste, and calories. Tony Sclafani discusses his recent work on this topic. Given that food preferences are believed to be important in obesity and eating disorders, it is important to demonstrate that different populations of human subjects (obese, normal-weight, eating-disordered) do indeed differ in their food preferences. Adam Drewnowski reviews his work, in which he has shown there are indeed such differences, and he also discusses cognitive factors that influence food preferences. Finally, Leann Birch discusses how children acquire food preferences. 